8 Chan JCN, Cockram CS, Nicholls MG, Cheung CK, Swaminathan R. Comparison of enalapril and nifedipine in treating non-insulin dependent diabetes associated with hypertension: one year analysis. BMJ 1992;305:981-5

9 Garg R, Yusuf S. Overview of randomized trials of angiotensinconverting enzyme inhibitors on mortality and morbidity in patients with converting enzyme inhibitors on mortality and morbidity in patients with
heart failure. Collaborative Group on ACE Inhibitor Trials. JAMA 1995;273:1450-6

10 Yusuf S, Peto R, Lewis J, Collins R, Sleight P. Beta blockade during and after myocardial infarction: an overview of the randomised trials. Progr Cardiovascular Dis 1985;27:335-71.

11 Heidenreich PA, Lee TT, Massie BM. Effect of beta-blockade on mortality in patients with heart failure: a meta-analysis of randomized clinical trials. JAm Coll Cardiol 1997;30:27-34.

12 Hypertension in Diabetes Study Group. HDS 3: prospective study of therapy in type 2 diabetic patients-efficacy of ACE inhibition and B-blockade. Diabet Med 1994;11:773-82.

13 Sawicki PT. Do ACE inhibitors offer specific benefits in the antihypertensive treatment of diabetic patients? Diabetologia 1998;41:598-602

14 Cooper ME. Pathogenesis, prevention and treatment of diabetic nephropathy. Lancet 1998:352:213-9.

15 Sawicki PT, Kaiser S, Heinemann L, Frenzel H, Berger M. Prevalence of renal artery stenosis in diabetes mellitus-an autopsy study. J Intern Med 1991;229:489-92.

16 Kumar A, Asim M, Davison AM. Taking precautions with ACE inhibitors. BMJ 1998;316:1921.
17 Andersson B, Caidahl K, diLenarda A, Warren SE, Goss F, Waldenstrom $\mathrm{A}$, et al. Changes in early and late diastolic filling patterns induced by long-term adrenergic beta-blockade in patients with idiopathic dilated cardiomyopathy. Circulation 1996;94:673-82.

18 Curb JD, Pressel SL, Cutler JA, Savage P, Applegate WB, Black H, et al. Effect of diuretic-based antihypertensive treatment on cardiovascular disease risk in older diabetic patients with isolated systolic hypertension. Systolic Hypertension in the Elderly Program Cooperative Research Systolic Hypertension in the E
Group. JAMA 1996;276:1886-92.

19 Ravid M, Savin H, Jutrin I, Bental T, Lang R, Lishner M. Long-term effect of ACE inhibition on development of nephropathy in diabetes mellitus type II. Kidney Int 1994;45(suppl):S161-4.

20 Lebovitz HE, Wiegmann TB, Cnaan A, Shahinfar S, Sica DA, Broadstone $\mathrm{V}$, et al. Renal protective effects of enalapril in hypertensive NIDDM: role of baseline albuminuria. Kidney Int 1994;45(suppl):S150-5.

21 Lewis EJ, Hunsicker LG, Bain RP, Rohde RD. The effect of angiotensin-converting-enzyme inhibition on diabetic nephropathy. $N$ Engl J Med 1993;329:1456-62.

22 Collins R, Peto R, MacMahon S, Herbert P, Fiebach NH, Eberlein KA, et al. Blood pressure, stroke, and coronary heart disease. Part 2. Short-term reductions in blood pressure: overview of randomised drug trials in their epidemiological context. Lancet 1990;335:827-38.

23 Collins R, MacMahon S. Blood pressure, antihypertensive drug treatment and the risks of stroke and of coronary heart disease. Br Med Bull 1994;50:272-98

(Accepted 20 August 1998)

\title{
Cost effectiveness analysis of improved blood pressure control in hypertensive patients with type 2 diabetes: UKPDS 40
}

\author{
UK Prospective Diabetes Study Group
}

Editorials by

Orchard and

Mogensen

Papers pp 703, 713

Members of the study group are

given at the end of the accompanying paper on $\mathrm{p} 703$.

This paper was prepared for publication by Maria Raikou, Alastair Gray, Andrew Briggs, Richard Stevens,

Carole Cull, Alistair McGuire, Paul Fenn, Irene Stratton, Rury Holman, and

Robert Turner.

Correspondence to: Dr Alastair Gray, Health Economics Research Centre, Institute of Health Sciences, Oxford University, Oxford OX3 7LF alastair.gray@ ihs.ox.ac.uk

Reprint requests to: UK Prospective Diabetes Study Group, Diabetes Research

Laboratories, Radcliffe Infirmary, Oxford OX2 6HE

BMJ 1998;317:720-6

\begin{abstract}
Objectives: To estimate the economic efficiency of tight blood pressure control, with angiotensin converting enzyme inhibitors or $\beta$ blockers, compared with less tight control in hypertensive patients with type 2 diabetes.

Design: Cost effectiveness analysis incorporating within trial analysis and estimation of impact on life expectancy through use of the within trial hazards of reaching a defined clinical end point. Use of resources driven by trial protocol and use of resources in standard clinical practice were both considered. Setting: 20 hospital based clinics in England, Scotland, and Northern Ireland.

Subjects: 1148 hypertensive patients with type 2 diabetes from UK prospective diabetes study randomised to tight control of blood pressure $(\mathrm{n}=758)$ or less tight control $(\mathrm{n}=390)$.
\end{abstract}

Main outcome measure: Cost effectiveness ratios based on $(a)$ use of healthcare resources associated with tight control and less tight control and treatment of complications and $(b)$ within trial time free from diabetes related end points, and life years gained. Results: Based on use of resources driven by trial protocol, the incremental cost effectiveness of tight control compared with less tight control was cost saving. Based on use of resources in standard clinical practice, incremental cost per extra year free from end points amounted to $£ 1049$ (costs and effects discounted at $6 \%$ per year) and $£ 434$ (costs discounted at $6 \%$ per year and effects not discounted). The incremental cost per life year gained was $£ 720$ (costs and effects discounted at $6 \%$ per year) and $£ 291$ (costs discounted at $6 \%$ per year and effects not discounted).
Conclusions: Tight control of blood pressure in hypertensive patients with type 2 diabetes substantially reduced the cost of complications, increased the interval without complications and survival, and had a cost effectiveness ratio that compares favourably with many accepted healthcare programmes.

\section{Introduction}

Hypertension in people with type 2 diabetes is associated with an increased risk of macrovascular complications. The systolic hypertension in the elderly programme showed the effectiveness of improved blood pressure in reducing the incidence of stroke and myocardial infarction in a diabetic subgroup of elderly patients (mean age 70 years) with type 2 diabetes, but no data on microvascular complications or on younger patients were available. ${ }^{1}$ The cost effectiveness of treatments based on antihypertensive drugs and education has been estimated for different general population groups, but these analyses have mainly been based on models and lack information on effectiveness and use of resources from long term trials, and none has considered hypertensive patients with type 2 diabetes. ${ }^{2-4}$ The hypertension in diabetes study reported in this paper, provides, for the first time, both the clinical information on microvascular and macrovascular complications, and the information on use of resources associated with treatment and managing complications, thereby allowing the cost effectiveness of tight blood pressure control in patients with type 2 diabetes to be assessed. ${ }^{5}$ 


\section{Methods}

\section{Patients, setting, and comparison}

In view of the high prevalence of hypertension (39\%) in the UK prospective diabetes study and of the uncertainties regarding its treatment, the hypertension in diabetes study, an embedded random allocation to less tight or tight control of blood pressure, was introduced in 1987. A total of 1148 hypertensive patients with type 2 diabetes (54\% male) were recruited from the study's patient population. Hypertension was defined as systolic blood pressure $\geqslant 160 \mathrm{~mm} \mathrm{Hg}$ or diastolic blood pressure $\geqslant 90 \mathrm{~mm} \mathrm{Hg}$ in patients not receiving antihypertensive treatment and blood pressures of $\geqslant 150 \mathrm{~mm} \mathrm{Hg}$ or $\geqslant 85 \mathrm{~mm} \mathrm{Hg}$ respectively in patients receiving hypertensive treatment.

The aim of the less tight control policy was initially to achieve blood pressure of $\leqslant 200 / 105 \mathrm{~mm} \mathrm{Hg}$, which was modified in 1992 to $<180 /<105 \mathrm{~mm} \mathrm{Hg}$ after publication of results of studies of elderly, non-diabetic hypertensive subjects. ${ }^{1}$ The aim of the tight control policy was to achieve blood pressure of $<150$ / $<85 \mathrm{~mm} \mathrm{Hg}$ using the angiotensin converting enzyme inhibitor captopril, $25 \mathrm{mg}$ twice daily increasing to $50 \mathrm{mg}$ twice daily, or the $\beta$ blocker atenolol, $50 \mathrm{mg}$ daily increasing to $100 \mathrm{mg}$ daily if required. If control criteria were not met other drugs were added.

The mean (SD) age of patients was 56.4 (8.1) years. Median duration of follow up was 8.4 years (range 0-10 years). The major clinical end points analysed were death or the development of diabetic complications, including coronary heart disease, cerebrovascular disease, amputations, laser treatment for retinopathy, cataract extraction, and renal failure. All analyses and comparisons were performed on the basis of intention to treat.

\section{Type of evaluation and perspective}

We performed an incremental cost effectiveness analysis in which we calculated the net costs and net effectiveness of tight control compared with less tight control and expressed these as a ratio. The main perspective of the economic evaluation was that of the healthcare purchaser. We analysed only direct health service costs. These costs covered the treatment costs for the policies of tight control and less tight control, visits to and tests at diabetic clinics, and the costs of treating diabetic complications.

\section{Resource data}

For each patient in the study, data were routinely collected on the dose of the two main antihypertensive drugs (captopril and atenolol); doses of nifedipine, diuretics, methyldopa, calcium channel blockers, vasodilators, and other antihypertensive drugs; doses of all drugs used for treating diabetes (insulin, sulphonylureas, metformin) and the number of home blood glucose tests; and whether the patient was taking anxiolytics and antidepressants, hormone replacement therapy, aspirin, or other drugs. When drug doses were not recorded, we replaced missing values by extrapolating from adjacent values for that patient.

The date and duration of any hospital admission were collected at each clinic visit. These were coded with ICD-9 and ICD-10 (international classification of diseases, ninth and 10th revisions) classifications for prime cause of admission, or OPCS- 4 codes for procedures undertaken. In addition, we maintained a separate record of all angiograms, angioplasties, or bypass grafting for coronary or peripheral vascular disease. All hospitalisations were also classified by two clinicians to one of 40 national standard specialty codes. We replaced missing values for hospital lengths of stay with the mean value for all patients in that specialty.

Data on use of non-inpatient healthcare resources were collected cross sectionally from all patients in the trial by means of a questionnaire distributed at routine clinic visits between January 1996 and September 1997 and by post to those who did not attend a clinic during that period. This questionnaire collected information on all home, clinic, and telephone contacts with general practitioners, nurses, chiropodists, opticians, dieticians, and eye and other clinics over the previous four months. We analysed these cross sectional data using multiple regression techniques to estimate for each patient annual use of non-hospital resources standardised for age, sex, body mass index, duration of diabetes, and time from a non-fatal end point related to diabetes.

\section{Costs}

We obtained unit costs for all resources used by trial patients from national statistics and from centres participating in the trial. For example, the daily cost in hospital by specialty was an average of the standardised financial returns of up to 241 hospitals across England. Table 1 summarises the main sources of information on unit costs. We combined these unit costs with the resource volumes to obtain a net cost per patient over the entire period of participation in the trial. We calcu-

\begin{tabular}{|c|c|c|}
\hline Item & $\begin{array}{c}\text { Unit cost } \\
\text { (1997 values) }\end{array}$ & Source \\
\hline $\begin{array}{l}\text { Specialist clinic visit in UK prospective } \\
\text { diabetes study }\end{array}$ & $£ 67.30$ & $\begin{array}{l}\text { Participating centres in UK prospective } \\
\text { diabetes study }\end{array}$ \\
\hline Drugs & Cost per item & British National Formulary 1997 \\
\hline "Standard practice" annual visits: & & See text for details \\
\hline \multicolumn{3}{|l|}{ Less tight control of blood pressure: } \\
\hline Diabetes control with diet and tablets & $£ 69.74$ & \\
\hline Diabetes control with insulin & $£ 157.79$ & \\
\hline \multicolumn{3}{|l|}{ Tight control of blood pressure: } \\
\hline Diabetes control with diet and tablets & $£ 149.39$ & \\
\hline Diabetes control with insulin & $£ 233.44$ & \\
\hline Inpatient day, by specialty: & & $\begin{array}{l}\text { Department of Health TFR2A costing returns } \\
\text { 1996-7. Mean for English NHS trusts }(n \leqslant 240)\end{array}$ \\
\hline General surgery & $£ 271$ & \\
\hline Ophthalmology & $£ 689$ & \\
\hline Ear, nose, and throat & $£ 511$ & \\
\hline Cardiothoracic surgery & $£ 464$ & \\
\hline Haemodialysis & $£ 24160$ & Average for 10 trusts \\
\hline Peritoneal dialysis & $£ 18140$ & Average for 11 trusts \\
\hline Retinal photocoagulation & $£ 655$ & Diabetes control and complications trial ${ }^{6 *}$ \\
\hline Other outpatient attendances & $£ 52.37$ & $\begin{array}{l}\text { Department of Health TFR2A costing returns } \\
\text { 1996-7. Mean for all English NHS trusts }\end{array}$ \\
\hline General practitioner: & & Netten and Dennett ${ }^{7}$ \\
\hline Surgery & $£ 10$ & \\
\hline Clinic & $£ 15$ & \\
\hline Home visit & $£ 30$ & \\
\hline Diabetes specialist nurse & $£ 22$ & Netten and Dennett ${ }^{7}$ \\
\hline Practice nurse (surgery) & $£ 6$ & Netten and Dennett ${ }^{7}$ \\
\hline
\end{tabular}

${ }^{*}$ Converted to £s (1997 values) by purchasing power parity. 
Table 2 Assumed annual number of "standard practice" visits for check up and tests, equivalent to level of care in hypertension in diabetes study, ${ }^{5}$ for policies of less tight control of blood pressure and tight control of blood pressure

\begin{tabular}{lcc} 
Visit & $\begin{array}{c}\text { Less tight } \\
\text { control }\end{array}$ & Tight control \\
\hline General practice nurse & 3 & 2 \\
\hline Specialist nurse & 0 & 2 \\
\hline General practice clinic & 1 & 2 \\
\hline Physician at a hospital diabetes clinic & 0 & 0 \\
\hline
\end{tabular}

lated mean net costs and associated 95\% confidence intervals per patient for each arm of the study. Costs are reported both undiscounted and in net present values by means of the $6 \%$ annual discount rate approved by the UK Treasury and a 3\% annual discount rate as recommended by the US Panel on Cost-Effectiveness. ${ }^{8}$ Discounting converts future costs and effects to present values, reflecting the conventional view that individuals put a higher value on resources used today than at some point in the future. All costs are reported in 1997 values of pounds sterling.

Protocol driven costs

All patients participating in the hypertension in diabetes study attended specialist clinics three or four times annually as set out in the study's trial protocol. However, in a non-trial standard practice setting it is likely that the frequency and type of visits would be different, particularly for the patients under less tight control of blood pressure. Therefore, in the main analysis we have costed visits for tight control or less tight control of blood pressure to reflect the likely pattern of standard clinical practice that would deliver the same levels of care as within the trial but excluding the protocol driven elements of cost. A similar approach has been adopted in other trial based economic evaluations of diabetes treatments such as the analysis of the diabetes control and complications trial. ${ }^{6}$ Table 2 outlines the likely pattern of standard practice for tight control and less tight control, based on clinical opinion in the hypertension in diabetes study. In this standard practice analysis, we replaced each patient's actual costs of annual trial visits by the estimated annual costs of visits in standard practice according to allocation. We also considered the costs of other patterns of care in sensitivity analyses.

\section{Outcomes}

Diabetes related end points were defined as in the clinical trial. ${ }^{5}$ We used Kaplan-Meier product limit estimates of time to these end points as reported in the hypertension in diabetes study ${ }^{5}$ to calculate years free from end points.

We estimated life expectancy beyond the end of the trial using a simulation model. The model expresses in parametric form hazard rates for events falling into three categories. In the cardiac category hazard rates for fatal events (myocardial infarction or sudden death) and non-fatal events (myocardial infarction or congestive heart failure) are considered to rise with age at diagnosis of diabetes and to rise even faster with duration of diabetes. ${ }^{9}$ A fixed proportion of first cardiac events are assumed to be fatal, and for a patient who has once experienced a non-fatal cardiac event there is assumed to be an increased hazard of fatal events. The effect of sex is also allowed for, with men having greater hazard. In the stroke category the hazards of fatal and non-fatal strokes are considered to rise with age at diagnosis of diabetes, duration of diabetes, and history of stroke in the same manner as for the cardiac category, but, because of lack of data, no effect of sex is modelled. In the third category all deaths not attributed to cardiac or cerebrovascular causes are considered, and the hazard for such a death is considered to rise with actual age (rather than age at diagnosis of diabetes). The model parameters were estimated from data from the hypertension in diabetes study and were fitted to the entire cohort.

Repeated trial cohorts were run through this model until all patients had died, and we recorded the mean life expectancy from randomisation to treatment to death in each arm. Each iteration of the model was preceded by a non-parametric bootstrap process in which treatment and control populations were sampled with replacement from the trial population to reflect the uncertainty in the observed results. The estimated gain in life expectancy resulting from the differences observed within the trial is conservative, since it is assumed that beyond the trial period the two groups have identical hazard rates. Moreover, as one moves beyond the end of the trial period the uncertainty in the estimated outcome increases because of the nature of the process of extrapolation. The full model will be described in detail in a future paper.

\section{Analysis}

We report all results as mean values with standard deviations, and mean differences in costs and effects with $95 \%$ confidence intervals. We calculated means and $95 \%$ confidence intervals for incremental cost effectiveness ratios using Fieller's method for estimating confidence intervals for ratios. ${ }^{10} 11$

Skewed data are often encountered in economic evaluations: although statistics such as the median are of interest descriptively, economic analysis is fundamentally concerned with mean values. ${ }^{12}$ When descriptive statistics indicated that skewness might be present, we performed 1000 bootstrap replications of the original data to test the robustness of the parametric assumptions concerning mean differences in cost. ${ }^{12} \mathrm{We}$ examined the effects of assumptions on our main results using sensitivity analyses. All data were analysed with sPSs 8.0 and Microsoft Excel 97.

\section{Results}

Table 3 shows the associated mean cost per patient over the duration of the study by category of cost and by allocation (also summarised in fig 1). The standard deviation of some of these mean costs suggested skewness in the data. We compared parametric confidence intervals for the cost differences with the bootstrap confidence intervals and found them to be robust. We therefore report parametric confidence intervals.

\section{Treatment costs}

Tight control of blood pressure increased the costs of antihypertensive drugs by an average of $£ 613$ (95\% confidence interval $£ 520$ to $£ 706$ ) compared with less 


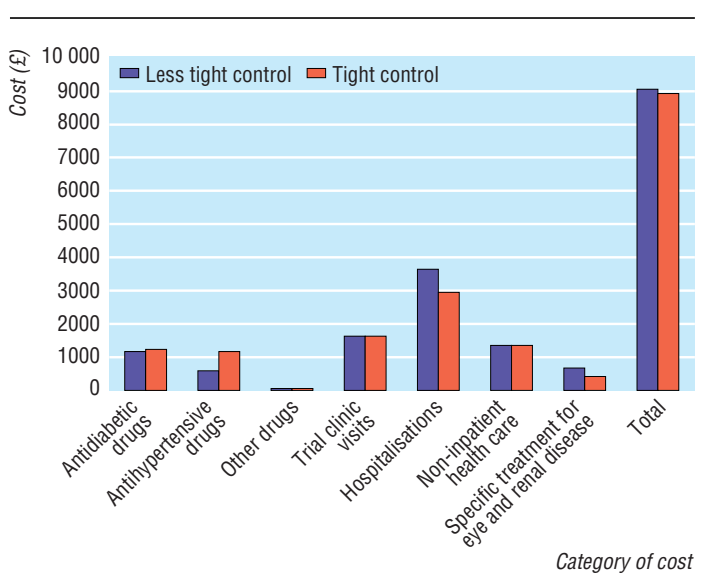

Fig 1 Mean cost per patient over median follow up of 8.4 years by category of cost and allocation to policy of less tight control of blood pressure or of tight control (based on use of resources driven by trial protocol, in 1997 values, and undiscounted)

tight control over median follow up of 8.4 years. There were no significant differences between patients assigned less tight control and those assigned tight control in the costs of antidiabetic drugs, other drug treatments, or trial visits to clinics. The total cost of treatment was $£ 3505$ per patient in the less tight control group and $£ 4245$ in the tight control group, an increase of $£ 740$ ( $£ 495$ to $£ 984$ ) over the duration of the trial.

When the costs of trial visits and tests were replaced by the estimates of the equivalent costs in standard clinical practice (as shown in table 2) total treatment costs became $£ 2289$ in the less tight control group and $£ 3417$ in the tight control group, an increase of $£ 1128$ (£913 to £1343).

\section{Complication costs}

The most costly complications were those involving hospitalisations. The mean cost of hospitalisation per patient in the group assigned less tight control of blood pressure was $£ 3603$ over the trial, compared with $£ 2930$ in the group assigned tight control, a difference of $£ 674$ ( $-£ 217$ to $£ 1564$ ). Thus a policy of tight control of blood pressure reduced the cost of complications requiring hospitalisation, although this failed to reach conventional levels of significance over the trial period $(\mathrm{P}=0.139)$.

Cross sectional analysis of responses to the questionnaire about non-inpatient healthcare use (standardised for age, sex, body mass index, and time from randomisation) indicated that a recent end point had a significant effect on non-inpatient costs, raising them on average by $£ 241$ in the first year, $£ 106$ in the second year, and $£ 80$ in the third year after the event. Thus, everything else being equal, a lower event rate in the group under tight control should be associated with lower non-inpatient costs. When calculated over the whole trial period, these costs added substantially to the total costs incurred in each arm, but there was no significant difference between the two arms. The costs associated with specific treatment of eye and renal disease (primarily retinal photocoagulation and renal dialysis) were slightly lower in the group assigned tight control, but this difference was not significant.
In total, therefore, tight blood pressure control was associated with a reduction in the cost of complications over the trial period of $£ 949$ ( $-£ 363$ to $£ 2261$ ) per patient.

\section{Total costs}

The increased costs of antihypertensive treatment in the group under tight control of blood pressure were offset by lower complication costs. Consequently, the net trial costs per patient of the two groups were not significantly different, at $£ 9085$ in the group under less tight control and $£ 8875$ in the group under tight control. Discounted at $6 \%$ per year to present values, these costs become $£ 7156$ with less tight control and $£ 7081$ with tight control.

These costs, however, reflect the use of resources driven by trial protocol. A more realistic approach is to replace these protocol driven elements by the likely pattern of visits that would produce equivalent care in a standard practice setting. On this basis the total cost of less tight control becomes $£ 7869$ per patient, compared with $£ 8048$ per patient with tight control, a difference of $£ 178$ ( $-£ 1187$ to $£ 1544$ ) in favour of less tight control. Discounted at $6 \%$ per year to present values, the total cost of less tight control becomes $£ 6145$ per patient, compared with $£ 6381$ per patient with tight control, a difference of $£ 237$ ( $-£ 809$ to $£ 1282$ ).

\section{Costs over time}

For the primary purpose of this economic evaluation, the costs reported were aggregated per patient over the whole trial period. However, the nature of the disease suggests that costs should increase over time, and this was indeed the case, as shown by the changes in mean undiscounted costs per patient by year (fig 2). It is clear that the costs of antihypertensive drugs, of hospitalisation, and total costs rose during the trial for both the patients assigned less tight control and those assigned tight control.

Table 3 Mean costs and mean cost differences for policies of less tight control of blood pressure and tight control of blood pressure by category of cost (1997 values, undiscounted unless stated otherwise)

\begin{tabular}{|c|c|c|c|}
\hline \multirow[t]{2}{*}{ Item } & \multicolumn{2}{|c|}{ Mean (SD) cost per patient (£) } & \multirow{2}{*}{$\begin{array}{c}\text { Mean }(95 \% \mathrm{CI}) \text { cost difference } \\
\text { per patient }(£)^{\star}\end{array}$} \\
\hline & Less tight control & Tight control & \\
\hline \multicolumn{4}{|l|}{ Costs of treatment } \\
\hline Antihypertensive drugs & $608(661)$ & $1221(929)$ & 613 (520 to 706$)$ \\
\hline Antidiabetic drugs & $1189(1471)$ & $1312(1663)$ & $122(-68$ to 311$)$ \\
\hline Other drugs & $43(61)$ & $41(57)$ & $-2(-10$ to 5$)$ \\
\hline Clinic visits & $1664(658)$ & $1671(650)$ & $7(-73$ to 87$)$ \\
\hline Total & 3505 (1894) & $4245(2188)$ & 740 (495 to 984$)$ \\
\hline \multicolumn{4}{|l|}{ Costs of complications } \\
\hline Hospital inpatient & $3603(7608)$ & $2930(6604)$ & $-674(-1564$ to 217$)$ \\
\hline Non-inpatient & $1304(647)$ & $1301(606)$ & $-3(-81$ to 74$)$ \\
\hline $\begin{array}{l}\text { Specific treatment for eye } \\
\text { and renal disease }\end{array}$ & 672 (6398) & $400(7516)$ & $-272(-1104$ to 559$)$ \\
\hline Total & $5580(10797)$ & $4630(10579)$ & $-949(-2261$ to 363$)$ \\
\hline \multicolumn{4}{|c|}{ Total costs of treatment and complications } \\
\hline Undiscounted & 9085 (11361) & 8875 (10 995) & -209 (-1584 to 1165$)$ \\
\hline $6 \%$ discount & $7156(8620)$ & $7081(8550)$ & $-74(-1126$ to 977$)$ \\
\hline $3 \%$ discount & 8024 (9839) & 7891 (9642) & $-133(-1329$ to 1062$)$ \\
\hline \multicolumn{4}{|c|}{ Total costs of treatment and complications in standard practice } \\
\hline Undiscounted & 7869 (11 285) & 8048 (10 929) & $178(-1187$ to 1544$)$ \\
\hline $6 \%$ discount & $6145(8572)$ & $6381(8506)$ & 237 (-809 to 1282) \\
\hline $3 \%$ discount & 6920 (9779) & 7132 (9589) & $212(-976$ to 1401$)$ \\
\hline
\end{tabular}

*Negative cost differences indicate cost savings associated with tight blood pressure control policy. 
Table 4 Time free from diabetes related end points and life years gained, from within trial effect of treatment, for policies of less tight control of blood pressure and tight control of blood pressure

\begin{tabular}{|c|c|c|c|}
\hline \multirow[t]{2}{*}{ Item } & \multicolumn{2}{|c|}{ Mean (SD) time per patient } & \multirow[b]{2}{*}{$\begin{array}{c}\text { Mean }(95 \% \mathrm{Cl}) \text { difference } \\
\text { per patient }\end{array}$} \\
\hline & $\begin{array}{l}\text { Less tight } \\
\text { control }\end{array}$ & Tight control & \\
\hline \multicolumn{4}{|c|}{ Within trial years free from end points: } \\
\hline Undiscounted & $7.61(3.61)$ & $8.16(3.52)$ & 0.54 (0.11 to 0.98$)$ \\
\hline $6 \%$ discount per year & $4.63(1.78)$ & $4.85(1.73)$ & $0.23(0.10$ to 0.44$)$ \\
\hline $3 \%$ discount per year & $5.88(2.55)$ & $6.24(2.48)$ & $0.35(0.05$ to 0.66$)$ \\
\hline \multicolumn{4}{|c|}{ Life years gained from within trial effect: } \\
\hline Undiscounted & $19.07(8.40)$ & $19.88(8.10)$ & $0.81(-0.21$ to 1.82$)$ \\
\hline $6 \%$ discount per year & $10.30(3.39)$ & $10.63(3.17)$ & $0.33(-0.08$ to 0.73$)$ \\
\hline $3 \%$ discount per year & $13.60(5.14)$ & $14.10(4.87)$ & $0.50(-0.11$ to 1.11$)$ \\
\hline
\end{tabular}

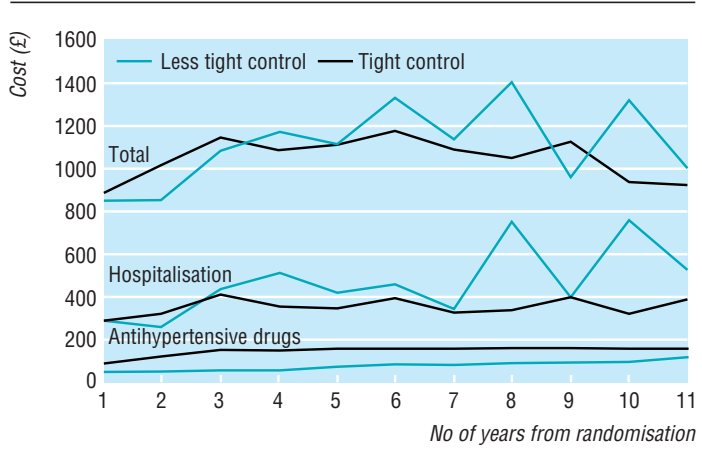

Fig 2 Mean cost per patient by year from randomisation and allocation to policy of less tight control of blood pressure or of tight control (based on use of resources driven by trial protocol, in 1997 values, and undiscounted)

\section{Outcomes}

The two main measures of effectiveness in this analysis were time free from diabetes related end points and life years gained. Table 4 shows that, based on the KaplanMeier product limit, the mean time to a diabetes related end point was 7.61 years in the group assigned less tight control and 8.16 years in the group assigned tight control, a mean difference of 0.54 (0.11 to 0.98$)$ years free from end points. Discounted at a $6 \%$ rate to present values, the difference in time to a diabetes related end point was 0.23 ( 0.10 to 0.44 ) years.

Based on the observed within trial effects of treatment, the modelled mean life expectancy from date of randomisation to the study was 19.07 years in the group assigned less tight control and 19.88 years in the group assigned tight control, a mean difference of $0.81(-0.21$ to 1.82$)$ years $(\mathrm{P}=0.118)$. Discounted at a $6 \%$ rate, the difference in life expectancy was 0.33 $(-0.08$ to 0.73$)$ years $(\mathrm{P}=0.112)$.

\section{Cost effectiveness}

Table 5 shows the cost effectiveness of tight blood pressure control compared with less tight control for the two main measures of outcome-time free from diabetic end points and life years gained. With regard to time free from end points, with both costs and effects discounted to present values at $6 \%$ per year, tight blood pressure control was cost saving when we considered the use of resources driven by the trial protocol. When we considered the use of resources in standard practice the cost per extra year free from end points was $£ 1049$ ( $-£ 4635$ to $£ 52373$ ), with both costs and effects discounted at $6 \%$. Discounting both costs and effects at a 3\% rate gives a cost per extra year free from end points of $£ 599$ ( $-£ 3400$ to $£ 13226$ ). Finally, discounting costs at $6 \%$ but without discounting effects, the cost per extra year free from end points was $£ 434(-£ 1633$ to $£ 6255$ ).

Uncertainty in cost effectiveness analysis exists on two levels: uncertainty in the estimated values of cost effectiveness and uncertainty about the maximum or ceiling cost effectiveness ratio that a decision maker would consider acceptable. One way of handling both levels of uncertainty is to construct a cost effectiveness acceptability curve, ${ }^{13}$ as shown in figure 3 . The $x$ axis shows a range of ceiling values for the incremental cost effectiveness ratio, and the $y$ axis shows the probability that the data are consistent with a true cost effectiveness ratio falling below any given ceiling ratio, based on the observed size and variance of differences in cost and effect in the trial. Thus, with costs and effects discounted at a $6 \%$ rate, there is a $33 \%$ probability that a policy of tight control of blood pressure

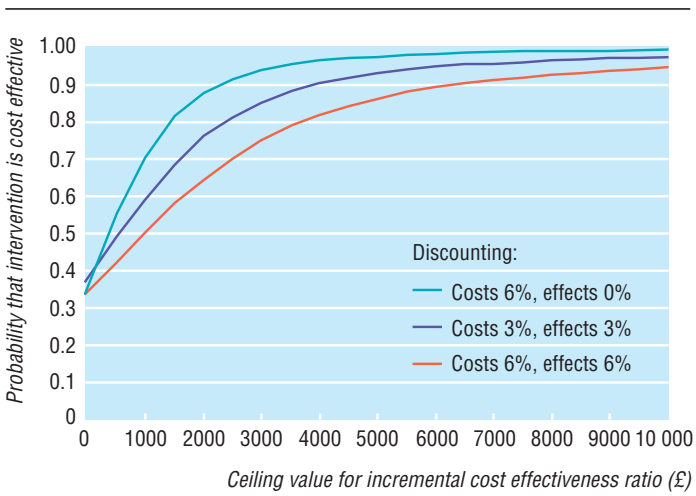

Fig 3 Cost effectiveness acceptability curves: probability that cost per extra year free from diabetes related end points is cost effective ( $y$ axis) as a function of decision maker's ceiling cost effectiveness ratio ( $x$ axis)

Table 5 Incremental cost effectiveness of policy of tight control of blood pressure compared with less tight control. Means $(95 \%$ confidence intervals) calculated with Fieller's method, costs based on 1997 values

\begin{tabular}{|c|c|c|c|c|}
\hline \multirow[b]{2}{*}{ Cost and outcome measure } & \multicolumn{2}{|c|}{ Costs and health effects discounted } & \multicolumn{2}{|c|}{ Costs only discounted } \\
\hline & $6 \%$ discount & $3 \%$ discount & $6 \%$ discount & $3 \%$ discount \\
\hline \multicolumn{5}{|c|}{ Time free from diabetes related end points: } \\
\hline Costs for standard practice & 1049 (-4635 to 52373$)$ & $599(-3400$ to 13226$)$ & 434 (-1663 to 6255) & $390(-2086$ to 6255$)$ \\
\hline Costs driven by trial protocol* & $-333(-10767$ to 23882$)$ & NA & NA & NA \\
\hline \multicolumn{5}{|l|}{ Life years gained ${ }^{\star \star}:$} \\
\hline Costs for standard practice & 720 & 422 & 291 & 261 \\
\hline Costs driven by trial protocol ${ }^{*}$ & -229 & NA & NA & NA \\
\hline
\end{tabular}

NA=Not applicable.

* Negative ratios costs indicate tight control is both more effective and cost saving.

**Estimated life years gained from tight control were not significant at $5 \%$ level (see table 4 ), hence $95 \%$ confidence intervals cannot be defined. 
would prove to be cost saving compared with a policy of less tight control, and a $50 \%$ probability that the cost per extra year free from end points lies above (or below) the point estimate of $£ 1049$ reported in table 5 . The upper limit to which the curve is tending corresponds to the probability that the tight control policy was less effective, in this case $2 \%$.

When the analysis was extended to life years gained from the within trial effects of treatment, as predicted by the simulation model, the cost per life year gained was $£ 720$ with both costs and effects discounted at a $6 \%$ rate. As the difference in effect was not significant at the $5 \%$ level, confidence intervals could not be calculated. However, it is again possible to express the results in the form of a cost effectiveness acceptability curve (fig 4). This shows that there is a $33 \%$ probability that a policy of tight control of blood pressure would prove to be cost saving per life year gained compared with a policy of less tight control, and a $50 \%$ probability that the cost per extra year free from end points lies above (or below) the point estimate of $£ 720$ reported in table 5 . The upper limit to which the curve is tending corresponds to the probability that the tight control policy is less effective, in this case $6 \%$.

The results for life years gained can also be interpreted in relation to previously published results for cost effectiveness. Figure 4 also shows the cost effectiveness of two other interventions (expressed in 197 values): cholesterol lowering in 59 year old men with a history of heart disease, as derived from the Scandinavian simvastatin survival study (£3200 per life year gained), ${ }^{14}$ and advice on lifestyle to 50 year old men to reduce cardiovascular risk, as reported from the Oxford and collaborators health check study ( $\$ 9500$ per life year gained). ${ }^{15}$ The figure indicates that there is an $83 \%$ probability that tight control of blood pressure for hypertensive patients with diabetes is more cost effective than secondary prevention of hypercholesterolaemia, and a $92 \%$ probability that it is more cost effective than lifestyle advice to lower cardiovascular risk.

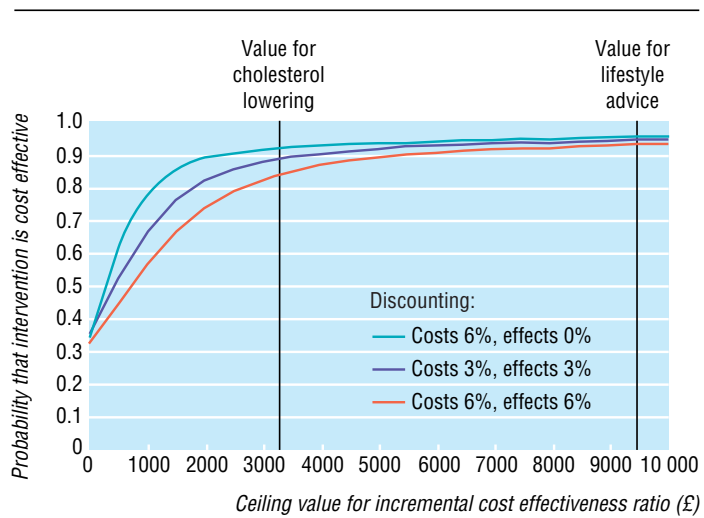

Fig 4 Cost effectiveness acceptability curves: probability that cost per life year gained from within trial effect of treatment is cost effective ( $y$ axis) as a function of decision maker's ceiling cost effectiveness ratio ( $x$ axis). Also shown is cost effectiveness of cholesterol lowering in 59 year old men with history of heart disease $^{14}$ and advice on lifestyle to 50 year old men to reduce cardiovascular risk ${ }^{15}$
Table 6 Sensitivity analyses on incremental cost effectiveness of policy of tight control of blood pressure compared with less tight control based on likely pattern of standard practice. Means $(95 \%$ confidence intervals) calculated with Fieller's method, all costs and health effects discounted at $6 \%$, costs based on 1997 values

\begin{tabular}{lc} 
Cost and outcome measure & Mean (95\% Cl) \\
\hline Time free from diabetes related end points: & \\
\hline Increased No of visits for tight control & $2164(-3638$ to 55 051) \\
\hline Increased No of visits for less tight control & $396(-11933$ to 21 358) \\
\hline Life years gained ${ }^{*}$ : & 1486 \\
\hline Increased No of visits for tight control & 272 \\
\hline Increased No of visits for less tight control &
\end{tabular}

*Estimated life years gained from tight control were not significant at $5 \%$ level (see table 4), hence $95 \%$ confidence intervals cannot be defined.

\section{Sensitivity analysis}

The cost and effect results reported here are derived from a large trial, and therefore most of the uncertainty surrounding the results can be expressed statistically. However, we performed sensitivity analysis on the likely pattern of standard practice for patients under tight control and those under less tight control, with incremental costs and effects discounted at $6 \%$ (table 6). If the numbers of visits to a specialist nurse and a general practitioner that are required to maintain tight blood pressure control are both increased from two to three annually, the cost per extra year free from end points rises from $£ 1049$ to $£ 2164$ ( $-£ 3638$ to $£ 55051)$ and the cost per life year gained rises from $£ 720$ to $£ 1486$. Alternatively, if the number of visits to a specialist nurse required for less tight control is increased from none to one annually, the cost per extra year free from end points falls from $£ 1049$ to $£ 396$ ( $£ 11933$ to $£ 21358)$ and the cost per life year gained falls from $£ 720$ to $£ 272$.

\section{Discussion}

This paper presents a comprehensive economic analysis of a tight blood pressure control policy in hypertensive patients with type 2 diabetes. It is based directly on information obtained from a clinical trial, the hypertension in diabetes study, and therefore uses data on the effectiveness and use of resources that are not prone to the sources of bias, confounding, and uncertainty that are likely to affect non-randomised studies. In addition, the long follow up in the hypertension in diabetes study allows the full range of costs arising from diabetic complications in patients assigned less tight control and tight control to be assessed empirically.

Diabetes is associated with a wide range of complications, and, consequently, it is important to adopt an outcome measure that captures all dimensions of health status. In this study we used within trial time free from diabetes related end points and life years gained from the within trial effect of treatment. Within trial time free from end points will be an underestimate of health gain, as the observed benefits of the intervention are likely to continue beyond the follow up period. We considered these longer term benefits by estimating life expectancy using a simulation model. We applied the parameter values of this model to both the group under tight control and the group under less tight control, making the conservative assumption of no continuing effect of tighter control beyond the trial 
period. The analysis presented here has not incorporated information on quality of life. Data from a cross sectional questionnaire completed towards the end of the study will allow cost utility analysis to be performed, and work on this aspect of the study will be reported in due course.

The within trial analysis shows that, compared with less tight control, tight control of blood pressure resulted in significant gains in time free from end points without a significant increase in total cost-with a point estimate of cost effectiveness ranging from $£ 390$ to $£ 1049$ per extra year free from end points depending on the discount rates used. Extending the analysis to the predicted effect of delaying diabetes related end points on life expectancy generates cost effectiveness ratios ranging from $£ 261$ to $£ 720$ per year of life gained. These point estimates suggest that tight control offers good value for money, but the attractiveness of this intervention to decision makers will depend on the uncertainty surrounding the point estimates, and on their willingness to pay for health gain.

The cost effectiveness acceptability curves presented in our analysis are a way of directly addressing both these issues. In particular, they convey information about the whole range of uncertainty rather than focusing exclusively on the $95 \%$ confidence limits. Although individual components of the cost effectiveness ratio may not be significant at conventional levels, acceptability curves allow us to take account of this while still determining the overall probability that the data are consistent with a cost effectiveness ratio less than some predetermined value. Thus, although our predictions of gains in life expectancy are not significant at the conventional $5 \%$ level, the acceptability curve shows that there is a greater than $90 \%$ chance that a true cost effectiveness figure for tight control of blood pressure would be below $£ 10000$ per year of life gained and a greater than $80 \%$ chance that a true cost effectiveness figure would be below $£ 3000$ per year of life gained.

Most economic evaluations of interventions in diabetes have been modelling exercises considering specific aspects of diabetic complications. ${ }^{16}{ }^{17}$ More general models of type 2 diabetes have not had access to long term data from trials on costs and effects of treatments. ${ }^{19}{ }^{19}$ Our analysis presents for the first time evidence suggesting that tight control of blood pressure for hypertensive patients with type 2 diabetes offers a cost effective means of reducing the risk of complications and improving health.

The cooperation of the patients and many NHS and non-NHS staff at the centres is much appreciated. We thank Philip Bassett, Valeria Frighi, Amanda Adler, and Ziyah Mehta for their contributions.

Funding: We thank the UK Department of Health, GlaxoWellcome, SmithKline Beecham, Pfizer, Zeneca, Pharmacia and Upjohn, Novo Nordisk, Bayer and Roche for grants for this health economics study. The main study was supported by grants from the UK Medical Research Council; the British Diabetic Association; the UK Department of Health; the US National Eye Institute; the US National Institute of Diabetes, Digestive and Kidney Disease in the National Institutes of Health, USA; the British Heart Foundation; Novo Nordisk; Bayer; Bristol-Myers Squibb; Hoechst; Lilly; Lipha; and Farmitalia Carlo Erba.

Conflict of interest: None.
Key messages

- Analysis of hypertensive patients with type 2 diabetes from the UK prospective diabetes study has shown that a policy of tight control of blood pressure substantially reduced both microvascular and macrovascular complications, conferring health benefit

- This economic analysis, conducted in conjunction with the clinical trial, shows that tight control of blood pressure in these patients substantially reduced the cost of complications, increased survival and the interval without complications, and had a cost effectiveness ratio that compares favourably with many accepted healthcare programmes

- On both clinical and economic grounds the use of a policy of tight control of blood pressure in hypertensive patients with type 2 diabetes is justified

1 SHEP Cooperative Research Group. Prevention of stroke by antihypertensive drug treatment in older persons with isolated systolic hypertension. Final results of the Systolic Hypertension in the Elderly Program (SHEP). JAMA 1991;265:3255-64.

2 Johannesson M, Agewall S, Hartford M, Hedner T, Fagerberg B. The costeffectiveness of a cardiovascular multiple-risk-factor intervention programme in treated hypertensive men. J Intern Med 1995;237:19-26.

3 Edelson JT, Weinstein MC, Tosteson ANA, Williams L, Lee TH, Goldman L. Long-term cost-effectiveness of various initial monotherapies for mild to moderate hypertension. JAMA 1990;263:407-13.

4 Nissinen A, Tuomilehto J, Kottke T, Puska P. Cost-effectiveness of the North Karelia hypertension program: 1972-1977. Med Care 1986;24: 767-80.

5 UK Prospective Diabetes Study Group. Tight blood pressure control and risk of macrovascular and microvascular complications in type 2 diabetes: UKPDS 38. BMJ 1998;317:703-13.

6 Diabetes Control and Complications Trial Research Group (DCCT). Lifetime benefits and costs of tight control therapy as practised in the diabetes control and complications trial. JAMA 1996;276:1409-15.

7 Netten A, Dennett J. Unit costs of community care. Canterbury: Personal Social Services Research Unit, University of Kent, 1998.

8 Gold M, Siegel J, Russell L, Weinstein M. Cost-effectiveness in health and medicine. Oxford: Oxford University Press, 1996.

9 Davis TM, Stratton IM, Fox CJ, Holman RR, Turner RC for the UK Prospective Diabetes Study (UKPDS) Group. Effect of age at diagnosis on diabetic tissue damage during the first 6 years of NIDDM. Diabetes Care 1997;20:1435-41.

10 Willan AR, O'Brien BJ. Confidence intervals for cost-effectiveness ratios: an application of Fieller's theorem. Health Econ 1996;5:297-305.

11 Chaudhary MA, Stearns SC. Estimating confidence intervals for cost-effectiveness ratios: an example from a randomized trial. Stat Med $1996 ; 15: 1447-58$.

12 Briggs A, Gray A. The distribution of health care costs and their statistical analysis for economic evaluation.J Health Serv Res Policy (in press).

13 Van Hout BA, Al MJ, Gordon GS, Rutten FF. Costs, effects and C/E-ratios alongside a clinical trial. Health Econ 1994;3:309-19.

14 Johannesson M, Jonsson B, Kjekshus J, Olsson AG, Pedersen TR, Wedel $\mathrm{H}$. Cost effectiveness of simvastatin treatment to lower cholesterol levels in patients with coronary heart disease. Scandinavian Simvastatin Survival Study Group. N Engl J Med 1997;336:332-6.

15 Wonderling D, Langham S, Buxton M, Normand C, McDermott C, Jones $\mathrm{L}$, et al. What can be concluded from the Oxcheck and British family heart studies: commentary on cost-effectiveness analyses. BMJ $1996 ; 312: 1274-8$.

16 Eckman MH, Greenfield S, Mackey WC, Wong JB, Kaplan S, Sullivan L, et al. Foot infections in diabetic patients. Decision and cost-effectiveness analyses. JAMA 1995;273:712-20.

17 Dasbach EJ, Fryback DG, Newcomb PA, Klein R, Klein BE. Cost-effectiveness of strategies for detecting diabetic retinopathy. Med Care 1991;29:20-39.

18 Eastman RC, Javitt JC, Herman WH, Dasbach EJ, Copley MC, Maier W, et al. Model of complications of NIDDM (I): model of construction and assumptions. Diabetes Care 1997;20:725-34.

19 Eastman RC, Javitt JC, Herman WH, Dasbach EJ, Copley MC, Maier W, et al. Model of complications of NIDDM (II): analysis of the health benefits and cost-effectiveness of treating NIDDM with the goal of normoglycemia. Diabetes Care 1997;20:735-44.

(Accepted 24 August 1998) 